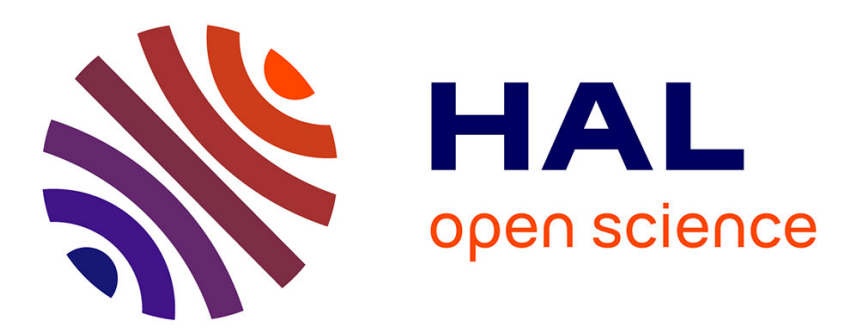

\title{
A model of the formation of stable nonpropagating magnetic structures in the solar wind based on the nonlinear mirror instability
}

Filippo Pantellini

\section{- To cite this version:}

Filippo Pantellini. A model of the formation of stable nonpropagating magnetic structures in the solar wind based on the nonlinear mirror instability. Journal of Geophysical Research Space Physics, 1998, 103 (A3), pp.4789 - 4798. 10.1029/97ja02384 . hal-03185757

\section{HAL Id: hal-03185757 \\ https://hal.science/hal-03185757}

Submitted on 30 Mar 2021

HAL is a multi-disciplinary open access archive for the deposit and dissemination of scientific research documents, whether they are published or not. The documents may come from teaching and research institutions in France or abroad, or from public or private research centers.
L'archive ouverte pluridisciplinaire HAL, est destinée au dépôt et à la diffusion de documents scientifiques de niveau recherche, publiés ou non, émanant des établissements d'enseignement et de recherche français ou étrangers, des laboratoires publics ou privés. 
An edited version of this paper was published by AGU. Copyright (1998) American Geophysical Union.

Pantellini, F. G. E. (1998), A model of the formation of stable nonpropagating magnetic structures in the solar wind based on the nonlinear mirror instability, J. Geophys. Res., 103( A3), 4789- 4798, doi:10.1029/97JA02384.

To view the published open abstract, go to https://doi.org/10.1029/97JA02384 


\title{
A model of the formation of stable nonpropagating magnetic structures in the solar wind based on the nonlinear mirror instability
}

\author{
Filippo G. E. Pantellini \\ Département de Recherche Spatiale, Observatoire de Paris-Meudon, Meudon, France
}

\begin{abstract}
A simple model for the formation of stable nonpropagating structures in a magnetized collisionless plasma is presented. The model describes the evolution of an electron-proton plasma from an initially spatially uniform, but unstable, configuration toward a final nonuniform and nonpropagating stable configuration. The model is based on the following hypothesis: (1) one-dimensionality and spatial periodicity, (2) cold electrons, (3) bi-Maxwellian protons as initial condition, (4) conservation of magnetic moment for all protons, (5) conservation of energy for magnetically non trapped protons, (6) spatial pressure balance, (7) evolved structure has a crenellated shape, (8) slow growth of the structure. Given these assumptions all the macroscopic properties of the plasma (density, pressure, and magnetic field) in the saturated state can be computed explicitly. The model shows that a spatially uniform and homogeneous plasma that is unstable against the linear mirror mode can form stable non propagating structures. Thus one can consider the model as a model for the nonlinear mirror instability where the magnetic trapping of protons in the low magnetic field region is the important saturation mechanism. A simple expression for the magnetic field saturation amplitude is found. The pressure balance, between high and low magnetic field regions, which is needed for the evolved structure to be a stable one, is obtained solely through betatron cooling of the trapped protons. Modification of the trapped protons energy due to the Fermi effect seems to be of secondary importance. The model predicts that the evolved structures are characterized by narrow and deep magnetic wells except in the case of very low magnetic pressure (ratio of thermal to magnetic pressure $\beta \gtrsim 10$ ) where the opposite situation becomes possible. This enforces the idea according to which the proton mirror instability is the driving mechanism for the formation of magnetic holes in high $\beta(z 1)$ plasmas.
\end{abstract}

\section{Introduction}

On a timescale of a minute or less, magnetic holes are the most commonly observed stable and nonpropagating structure in the solar wind. Even though a rigorous definition of magnetic hole has not yet been given, such structures are generally identified as more or less deep dropouts of the magnetic field. , The magnetic field intensity within a hole can be as low as $10 \%$ of the out of hole magnetic field. Typical durations are in the interval ranging from a few seconds up to 1 or $2 \mathrm{~min}$ [Winterhalter et al., 1994]. Magnetic holes have been observed in the Earth magnetosheath [e.g., Kaufmann et al., 1970; Tsurutani et al., 1982], in the free solar wind [Turner et al., 1977; Fitzenreiter and Burlaga, 1978; Klein and Burlaga, 1980; Winterhalter et al., 1994], mostly in the vicinity of stream interfaces, and at comets [Russell et al., 1987; Glassmeier et al., 1993]. The total pressure

Copyright 1998 by the American Geophysical Union.

Paper number 97JA02384.

0148-0227/98/97JA-032384\$09.00 (particle + field) inside a magnetic hole is generally believed to be balanced by the pressure of the surrounding plasma even though observations do not seem to be very conclusive on that point. However, it is clear that if the pressure of a nonpropagating magnetic hole is not balanced by the pressure of the surrounding plasma, it must be unstable and either collapse or explode. As we are only concerned with stable structures, we shall consider the case of nonpropagating magnetic holes that are in pressure balance with the surrounding plasma.

Magnetic holes are mainly observed in high $\beta$ (the ratio of thermal pressure to magnetic pressure) plasmas with $\beta z 1$. Thus, soon after the very first observations of magnetic holes, the proton mirror instability, which is driven by an anisotropic thermal pressure of the protons such that $p_{\perp} / p_{\|}>1$ (subscripts $\perp$ and $\|$ refer to the local magnetic field direction), has been proposed to be the basic mechanism leading to their formation. In fact, in a high $\beta$ plasma, the proton temperature anisotropy required for instability is rather weak, that is $T_{\perp} / T_{\|}>1+1 / \beta_{\perp}$ [e.g., Southwood and Kivelson, 1993]. The mirror instability has some other particularities that make it a privileged candidate to fit into 
a scenario of magnetic holes formation, the nonpropagating nature of the mode and the anticorrelation of density and magnetic field being two other important reasons.

Since the late 1950s the theory of the linear proton mirror instability has been discussed by a large number of authors [see Pantellini and Schwartz, 1995, and references therein]. However, the underlying physical processes, implicit in the early treatments of the instability [e.g., Barnes, 1966; Tajiri, 1967], have been elucidated only recently by Southwood and Kivelson [1993]. The new important aspect which has been pointed out in the Southwood and Kivelson [1993] theory is that the mirror instability is one where the resonant particles, that is, particles with small velocities parallel to the magnetic ficld, play an important role. This has been the strongest demonstration of the fact that the mirror instability is based on essential kinetic effects and that, as a consequence, fluid theories do not lead to a correct understanding of the instability.

The rather speculative hypothesis according to which the mirror instability is responsible for the formation of magnetic holes has been consolidated after the identification of mirror mode waves in planetary magnetosheaths [e.g., Tsurutani et al., 1982; Hubert et al., 1989; Lacombe et al., 1992; Anderson and Fuselier, 1993; Violante et al., 1995]. The hypothesis has become even less speculative since Winterhalter et al. [1994] could show that trains of closely spaced magnetic holes are often observed in marginally mirror mode stable plasmas and also that proton distribution functions observed within holes are sometimes similar to distributions seen in numerical simulations of mirror mode waves [e.g., McKean et al., 1993]. From the theoretical point of view the most stringent proof of the causal relationship between mirror instability and magnetic holes has been given recently in a paper on the nonlinear mirror instability by Kivelson and Southwood [1996]. The theory of Kivelson and Southwood [1996] indicates that if one admits that particles trapped in the magnetic wells, created by the instability, do undergo a mixturc of both betatron deceleration, due to the locally decreasing field intensity, and Fermi acceleration, due to the relative motion of the mirror points, then the mirror instability may evolve toward a saturated state characterized by deep and narrow holes in the magnetic field profile. The Kivelson and Southwood [1996] theory is based on the fact that the evolved structure must satisfy the pressure balance condition. This is only possible if one allows for substantial cooling of the trapped proton population. However, the amount of cooling needed to saturate the instability depends on the spatial and temporal evolution of the magnetic field. Unfortunately, the spatial and temporal dependence of the magnetic field is not given by the Kivelson and Southwood [1996]theory.

Our approach is similar to the one of Kivelson and Southwood [1996] with the important difference that we restrict the analysis to a particularly simple profile of the evolved spatial structure which we expect to be a rough approximation of the real structure of the saturated nonlinear mirror instability (see Figure 1). Following Kivelson and Southwood [1996], our model is based on a spatial pressure balance condition between the high and low magnetic field re- gions. The pressure balance condition includes the contribution from both magnetically trapped and nontrapped (circulating) protons. The advantage of restricting the evolved magnetic field profile to the simple shape shown in Figure 1 is that the model can be calculated easily throughout. In particular, we will show that for one given initial plasma condition, which is fully specified by two parameters (the proton pressure anisotropy and the plasma $\beta$ ), there is at most one final stable plasma configuration. This is completely different than in the Kivelson and Southwood [1996] theory where the emphasis is put on the qualitative description of the cooling of the trapped proton population needed to reach a given final state which may be taken from observations. The main problem with that stems from the fact that it is extremely difficult, not to say impossible, to specify a plasma cquilibrium consistent with an observed non uniform magnetic field profile. Given the strong uncertainties on the final state, it is virtually hopeless to determine how such a state has been obtained from an initially unstable (and a priori unknown) configuration.

In our approach we start from an easy to define initial state and compute the final state using a number of assumptions that make the problem simpler than the one addressed by Kivelson and Southwood [1996] at the expense of a loss of generality. We shall see that even in the framework of this simplified theory a final stable configuration can be obtained in most cases. We shall argue that the detailed motion of the trapped particles is unimportant in the saturation process. We shall also show that the model leads naturally to the formation of deep and narrow magnetic holes (observed) rather than the opposite situation characterized by large and shallow holes (rarely observed). In the latter case one would no longer define these structures as magnetic holes but rather as "magnetic monoliths."The qualitative agreement between the results of our model and the observations suggests that the former includes most of the important physics necessary to generate the observed nonpropagating structures.

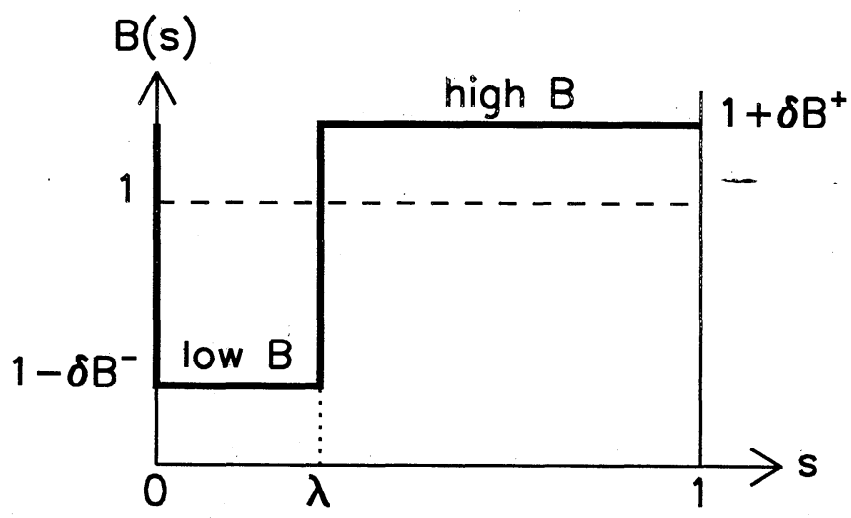

Figure 1. Magnetic field profile for $t>0$. At $t=0$ the field is a constant $B_{0}=1$ over the whole domain $s \in[0,1]$. The model is spatially periodic with period 1 . Together with Faraday's equation, periodicity implies that the average magnetic field is equal to $B_{0}$ at any time. Thus $\lambda, \delta B^{-}$ and $\delta B^{+}$are related through (2). Note that all other quantities appearing in the model (number density, pressure, etc.) are always taken to be constant in the intervals $[0, \lambda]$ (low- $B$ field) and $[\lambda, 1]$ (high- $B$ field). 
We conclude by noting that the first attempt to describe the nonlinear evolution of the mirror instability has been proposed, long ago, by Shapiro and Shevchenko [1964]. Their approach was based on quasi-linear theory. However, as pointed out by Kivelson and Southwood [1996], quasi-linear theory is most probably inappropriate in the treatment of the mirror instability except in the case of very weak growth. The main reason is that quasi-linear theory predicts a spatially uniform final state while simulations [e.g., McKean et al., 1992; McKean et al., 1993], as well as the above cited observations, indicate that the evolved state of the mirror instability is generally characterized by strong spatial variations.

\section{Basic Assumptions of the Model}

We start from the postulate that both the particle trapping and a spatial pressure balance condition are the main ingredients in a theory of the evolution of the nonlinear mirror instability. Based on these two main assumptions, we construct a simple model for the nonlinear evolution of the mirror instability.

We consider a model where, at any given time $t$, all quantities (c.g., density, pressure, magnetic field, ...) depend on one spatial dimension only. As in linear theory, it is the motion of the particles along the magnetic field lines which controls the evolution of the system [Kivelson and Southwood, 1996] so that the relevant spatial dimension is along the field line. We restrict the model to a two species proton-electron plasma. This restriction is a minor one as heavy ions, which are present in the solar wind, do not affect significantly the mirror instability [Price et al., 1986]. For simplicity we assume that the electrons are cold so that they do not produce any longitudinal electric field [cf. Pantellini and Schwartz, 1995]. Our purpose is to predict the final state of the plasma given an unstable uniform initial equilibrium. Thus at $t=0$ the plasma is taken to be spatially uniform, the protons being distributed according to a bi-Maxwellian

$$
f_{0}=\frac{n_{0} \pi^{-3 / 2}}{v_{T \perp}^{2} v_{T \|}} \exp \left[-\frac{v_{0 \|}}{v_{T \|}^{2}}-\frac{v_{0 \perp}}{v_{T \perp}^{2}}\right]
$$

where $v_{T_{\perp}} \equiv\left(2 k_{B} T_{\perp} / m\right)^{1 / 2}$ and $v_{T_{\|}} \equiv\left(2 k_{B} T_{\|} / m\right)^{1 / 2}$ designate the proton thermal velocity perpendicular and parallel to the uniform magnetic field $B_{0}$. Here $m$ is the proton mass, $k_{B}$ is the Boltzmann constant and $n_{0}$ is the proton number density.

Hereinafter we adopt the following normalizations: $n_{0}=$ $1, B_{0}=1$ and $v_{T \|}=1$ the plasma at $t=0$ being completely specified by the dimensionless parameters $\beta_{\|} \equiv$ $2 \mu_{0} k_{B} n_{0} T_{\|} / B_{0}^{2}$ and the ratio $R \equiv T_{\|} / T_{\perp}$. We further assume that the system is spatially periodic, with period 1 , and that at any time $t>0$ the magnetic field is constant in the intervals $[0, \lambda]$ and $[\lambda, 1]$ (see Figure 1). We shall denote quantities characterizing the high magnetic field region by a plus superscript and quantities characterizing the low magnetic field region by a minus superscript. Subscript 0 denotes time level $t=0$ whenever this indication is needed to avoid ambiguity. We also note that the scale of length of the sys- tem does not need to be specified as only relative dimensions will be used. Moreover, since the model is one dimensional and periodic, from Faraday's equation $\partial \mathbf{B} / \partial t=-\nabla \times \mathbf{E}$ it follows that the average magnetic field must be constant in time. Thus from Figure 1 one deduces that $\lambda, \delta B^{+}$and $\delta B^{-}$ are related to each other through

$$
(1-\lambda) \delta B^{+}=\lambda \delta B^{-} \text {. }
$$

Hereinafter we will sometimes use the notation $B^{ \pm} \equiv 1 \pm$ $\delta B^{ \pm}$.

\section{Density and Pressure in the High Magnetic Field Region}

In order to compute the number density $n^{+}$and the perpendicular particle pressure $p_{\perp}^{+}$in the high magnetic field region, we note that in a slowly growing magnetic structure nearly all particles do conserve energy. Only a small number of particles for which the time needed to cross the whole system is longer than the typical growth time of the magnetic structure do not conserve energy. These particles, called resonant particles in linear theory, gain or lose energy depending on whether the local field is growing or decreasing (betatron effect) and have been described in detail by Southwood and Kivelson [1993]. Using the fact that the particles' energy $m\left(v_{\perp}^{2}+v_{\|}^{2}\right) / 2$ and the particles' first adiabatic invariant $\mu=m v_{\perp}^{2} /(2 B)$ are both conserved one finds that only particles satisfying the condition

$$
v_{0 \|} / v_{0 \perp}>\sqrt{\delta B^{+}}
$$

can reach the high magnetic field region corresponding to the interval $[\lambda, 1]$. Thus we call circulating particles those particles which do satisfy to the inequality (3) and trapped particles those which do not. The two populations are separated by the trapping boundary, or separatrix, defined by $v_{0\|\|} / v_{0 \perp}=\sqrt{\delta B^{+}}$. It is obvious that trapped particles are located in the interval $[0, \lambda]$, whereas the circulating particles are distributed over the whole available space $[0,1]$.

Taking into account that $f$ is constant along particle trajectories and that energy and magnetic moment of the circulating particles are both conserved, it is easy to compute the number density $n^{+}$and the particle pressure $p_{\perp}^{+}$in the high magnetic field region. Thus for $n^{+}$we have

$$
\begin{aligned}
& n^{+}=\int_{D_{t}^{+}} f^{+}\left(v_{\|}, v_{\perp}, t\right) d v^{3} \\
& n^{+}=\int_{D_{t}^{+}} f^{+}\left(v_{\|}, v_{\perp}, t\right) J^{+} d v_{0}^{3} \\
& n^{+}=\int_{D_{0}} f_{0}\left(v_{0 \|}, v_{0 \perp}\right) J^{+} d v_{0}^{3} \\
& n^{+}=\int_{0}^{\infty} 2 \pi v_{0 \perp} d v_{0 \perp} \int_{v_{0 \|} \mid>v_{0 \perp} \sqrt{\delta B^{+}}} f_{0}\left(v_{0 \|}, v_{0 \perp}\right) \\
& n^{+}= \\
& J^{+}\left(B^{+}, v_{0 \|}, v_{0 \perp}\right) d v_{0 \|} \\
& R+\delta B^{+}
\end{aligned}
$$


where $D_{t}^{+}$denotes the domain in velocity space occupied by the circulating particles spatially located in the high magnetic field region at time $t$, while $D_{0}$ denotes the domain in velocity space occupied by the same particles at $t=0$. $\mathrm{J}^{+}$represents the Jacobian giving the variation of the velocity space volume element $d v_{0}^{3}=2 \pi v_{0 \perp} d v_{0 \perp} d v_{0 \|}$ during the time interval $t$. The Jacobian can be computed from the equations of conservation for energy and magnetic moment. This leads to

$$
J^{+}\left(B^{+}, v_{0 \|}, v_{0 \perp}\right)=\frac{\left|v_{0 \|}\right| B^{+}}{\left(v_{0 \|}^{2}-v_{0 \perp}^{2} \delta B^{+}\right)^{1 / 2}} .
$$

Equation (4) shows that the density in the high-field region is always smaller than the initial density value $n_{0}=1$, and that for small amplitudes $\delta B^{+} \ll 1$, the result $n^{+}=$ $1+\delta B^{\prime}(1-1 / R)$, known from the linear theory of the mirror instability, is recovered [Southwood and Kivelson, 1993]. This makes clear that our model is effectively a model for the nonlinear mirror instability.

The mappings of $D_{0}$ resulting from the motion of the circulating particles to both high and low magnetic field regions are illustrated in Figure 2. As $D_{0}$ extends to infinity, only the mapping of a part of the distribution function $f_{0}$ (shaded region), delimited by an arbitrary contour level, is actually shown in the figure. The fact that for $\delta B^{+} \rightarrow \infty$ one obtains a nonvanishing asymptotic density value $n^{+} \rightarrow R$ can be easily understood from Figure $2 b$. Since by definition the energy of the circulating particles is conserved, particles do move on circles in velocity space when being mapped from the initial contour, corresponding to the distribution at $t=0$ to the new contour at time $t>0$. Thus in the limit $\delta B^{+} \rightarrow \infty$ the major half-axis of the half-ellipse $D_{t}^{+}$ shrinks to the same values as the minor half-axis, that is $D_{t}^{+}$ becomes a nonvanishing half circle. It is also clear from the figure that in the same way $f^{+}\left(v_{\|}, v_{\perp}, t\right)$ must tend toward an isotropic Maxwellian distribution with temperature $T=T_{\|}$since it results from the mapping, along circles, of the points $f_{0}\left(v_{0 \|}, 0\right)$. The integration over the resulting isotropic distribution leads to the above asymptotic result $n^{+}=R$.

The question mark in Figure 2c illustrates the fact that, as we shall show below, the motion of the trapped particles in velocity space does not have to be known in order to compute the model throughout. The only assumption concerning the trapped particles is that their magnetic moment is conserved. However, the energy of a particle trapped near the scparatrix has to be conserved if one asks for the distribution $f$ to be continuous there. We shall point out that this has not to be true (and is certainly not true in general!) for all other trapped particles. The complicated behavior of the trapped particles, which is implicit in our model, is schematically illustrated in Figure 3 . The top panel (Figure $3 a)$ shows that circulating particles are thought to be energy conserving since the time these particles spend in the low, or high, field region is assumed to be small compared to the typical growth time of the magnetic structure. Similarly, a particle that has just been trapped, that is, a particle with mirror points at a magnetic field strength close to $B^{+}$, has spent most of the time as circulating particle, so that its energy did not have time to depart significantly from the initial value. Particles that have mirror points corresponding to a field strength significantly less than $B^{+}$have been trapped during a time which is comparable to the typical growth time. Two effects can affect the energy of these particles. (1) The particle loses energy due to fact that it sees a decreasing magnetic field (Figure $3 \mathrm{~b}$ ). This is the so-called be-
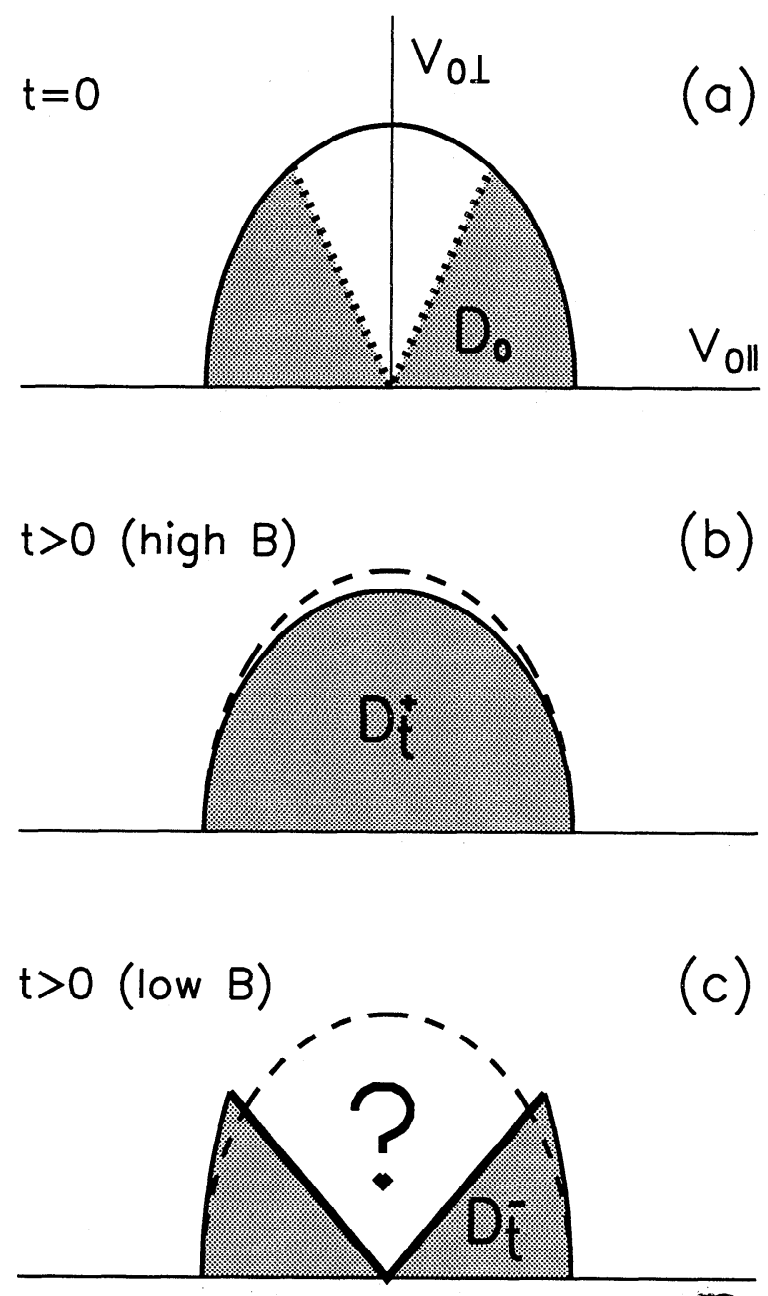

Figure 2. Mapping of the velocity distribution function of circulating particles to both high- and low- $B$ field regions. (a) A particular contour level $c_{l}$ of the distribution function $f_{0}\left(v_{0 \|}, v_{0 \perp}\right)$ is shown. The interior of the half-ellipse corresponds to the domain with $f_{0}>c_{l}$. The shadowed domain corresponds to the circulating particles for which the inequality (3) is true, given a certain $\delta B^{+}$. The remaining sector of the ellipse corresponds to the particles which arc trapped for this same $\delta B^{+}$. (b, c) Mapping of the circulating particles to high- and low-field regions with the original contour being represented by the dashed line. Obviously, there are no trapped particles at magnetic field maximum (Figure $2 \mathrm{~b}$ ). As the model only requires that trapped particles conserve magnetic moment $\mu$, but not energy, their velocity distribution is not known for $t>0$, thus the question mark in Figure 2c. 


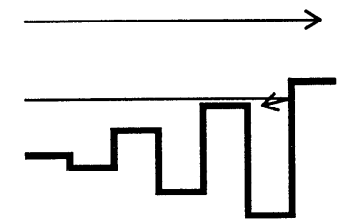

(a)

energy conserving

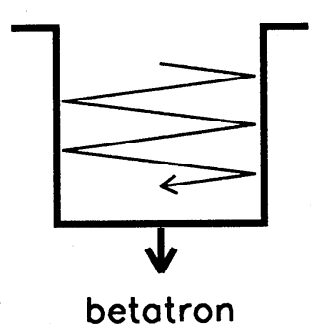

(b)

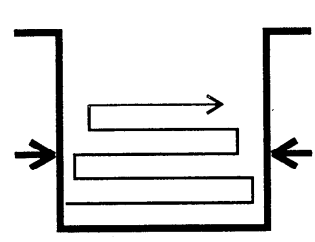

(c)

Fermi

Figure 3. Time dependence of the energy for particles in the model. (a) A circulating and a freshly trapped particle are shown. Both particles do approximately conserve energy since the average magnetic field the particles see during the typical growth time of the structure is roughly constant. A particle that is trapped during a time which is comparable to the time of evolution of the magnetic field may change its energy due to two distinct mechanisms illustrated in Figures $3 \mathrm{~b}$ and $3 \mathrm{c}$. (b) The trapped particle loses energy at the rate $\mu \partial B / \partial t$ as it experiences a constantly decreasing magnetic field (betatron deceleration), while (c) the trapped particle gains energy as the magnetic hole contracts and the magnetic mirror points move toward each other (Fermi acceleration).

tatron deceleration. It affects the perpendicular velocity of the particle. (2) The particle gains (loses) energy because of the converging (diverging) mirror points (Figure $3 \mathrm{c}$ ). This is the so-called Fermi acceleration (deceleration). It affects the particle's parallel velocity. We shall show below that only the betatron effect does play a role in our model and that the Fermi effect is of secondary importance in the saturation of the mirror instability.

As announced, we may now compute the particle pressure $p^{+}$at magnetic field maximum in the same way as we did for the number density $n^{+}$. Keeping in mind that $v_{\perp}^{2}=$ $v_{0 \perp}^{2} B^{+}$for the particles circulating in the high-field region, we obtain

$$
\begin{aligned}
& p_{\perp}^{+}=\int_{D_{0}} f_{0}\left(v_{0 \|}, v_{0 \perp}\right) v_{0 \perp}^{2} B^{+} J^{+} d v_{0}^{3} \\
& p_{\perp}^{+}=R\left(\frac{B^{+}}{R+\delta B^{+}}\right)^{2} .
\end{aligned}
$$

It should be noted that $n^{+}$and $p_{\perp}^{+}$do not depend on $\delta B^{-}$ and $\lambda$. This is a consequence of the fact that the equations of motion for particles reaching the high-field region only depends on $\delta B^{+}$. This is not true for particles located in the magnetic wells. For example, circulating particles in the low-field region do satisfy to the same inequality (3) as do the circulating particles in the high magnetic field region, but their perpendicular velocity depends on $B^{-}$since $v_{\perp}^{2}=$ $v_{0 \perp}^{2} B^{-}$. Thus their contribution to the density and pressure depend on both $B^{+}$and $B^{-}$.

\section{High-Field Saturation Level}

In the previous section we have computed the density and the particle pressure in the high magnetic field region for a given magnetic field amplitude $\delta B^{+}$. In this section we shall compute the saturation amplitude $\delta B_{\text {sat }}^{+}$in the case of an unstable plasma.

Now from the previous section we already know that, for a given value of the field perturbation $\delta B^{+}$the total perpendicular plasma pressure is given (in normalized units) by

$$
p_{\perp, \mathrm{tot}}^{+}=R\left(\frac{B^{+}}{R+\delta B^{+}}\right)^{2}+\frac{\left(B^{+}\right)^{2}}{\beta_{\|}} .
$$

The important fact about this expression is that it does not depend on the characteristics of the low magnetic field region. It is therefore clear that the saturation amplitude $\delta B_{\text {sat }}^{+}$ must depend on the initial plasma parameters $\beta_{\|}$and $R$ only. Another important point about (7) is that for small values of $\delta B^{+}$the total pressure and the magnetic pressure vary in antiphase provided $R^{-1}-1-R / \beta_{\|}>0$, which is precisely the instability condition for the linear mirror instability. In fact, (7) is the finite amplitude equivalent of the magnetohydrodynamic response of a bi-Maxwellian plasma to a small, and slowly varying, compressional perturbation $\delta B^{+}$, which reads

$$
\delta p_{\perp, \text { tot }}^{+}=2 p_{0 \perp, \text { tot }}^{+}\left(1-R^{-1}\right) \delta B^{+} / B_{0}
$$

and is the base of any theory of the linear mirror instability [e.g., Hasegawa, 1969; Southwood and Kivelson, 1993]. We now postulate that even in the nonlinear regime the instability keeps on growing as long as the total pressure and the magnetic field pressure vary in antiphase. By setting $\partial p_{\perp, \text { tot }}^{+} / \partial B^{+}=0$ one computes directly the limiting value $\delta B_{\text {sat }}^{+}$beyond which the total pressure and the magnetic field pressure are both growing. This leads to a simple expression for the saturation amplitude

$$
\frac{\delta B_{\mathrm{sat}}^{+}}{B_{0}}=\left[\beta_{\|} R(1-R)\right]^{1 / 3}-R .
$$




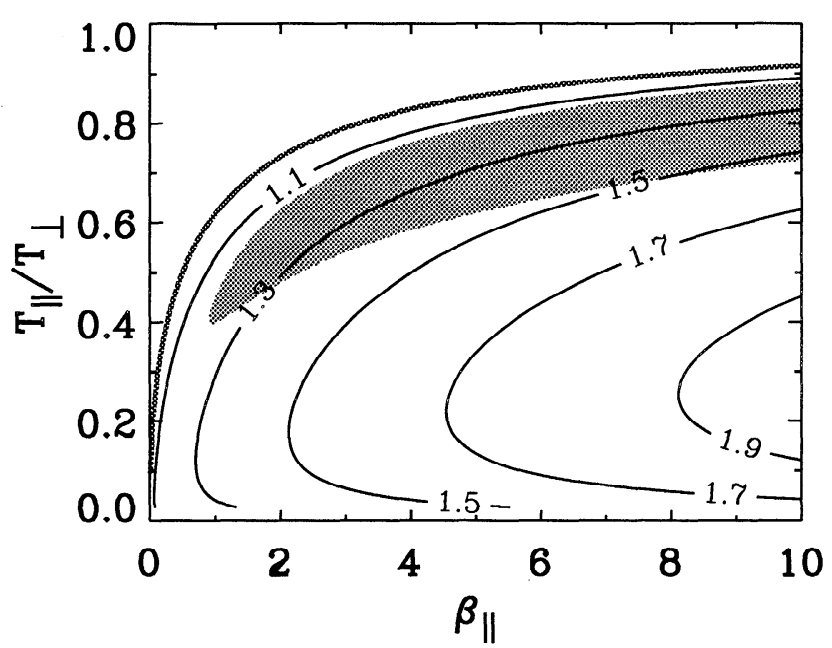

Figure 4. Contours of $B_{\text {sat }}^{+}$computed from (8). The upper thick contour corresponds to the stability threshold for the linear mirror mode. The meaning of the shaded region will be discussed in relation to Figure 5.

We note that (8) gives the saturation peak value for an arbitrary magnetic field profile which may be different from the one shown in Figure 1. This is a consequence of the fact that no reference to the actual magnetic field profile has been made in deriving equation (8). An example: for $\beta_{\|}=1$ and $R=0.5,(8)$ predicts a saturation amplitude $\delta B_{\text {sat }}^{+}=0.13$. The same value has been obtained numerically by Kivelson and Southwoo using a sinusoidal field profile (compare Figure 3 of Kivelson and Southwood [1996]).

A contour plot for $B_{\text {sat }}^{+}\left(\beta_{\|}, R\right)$ is shown in Figure 4. The thick curve corresponds to the value $B_{\text {sat }}^{+}=1$ which separates the mirror stable (above the curve) from the mirror unstable domain. The figure shows that for reasonably weak anisotropies of the proton distribution the saturation amplitude increases with $\beta_{\|}$and $T_{\perp} / T_{\|}$. For strong anisotropies $R \rightarrow 0$ we do not expect the model to be valid any longer as the growth rate would then be so strong that most of the circulating particles would not conserve energy which, of course, is against one of the basic assumptions of the model.

\section{Saturation in the Low-Field Region and Overall Structure at Saturation}

The explicit shape of the magnetic field profile has to be considered in the derivation of the plasma conditions in the low magnetic field region. Our purpose is to determine $\delta B^{-}$ and $\lambda$, which are defined in Figure 1, such that the resulting structure is a stable equilibrium. Stability of the structure requires that the total pressure is the same at field minimum and field maximum [Kivelson and Southwood, 1996]. In this section we shall use a pressure balance condition in order to compute the complete macroscopic state of the plasma at saturation.

The density ratio $\eta \equiv n^{-} / n^{+}$(where the density $n^{-}$includes both trapped and circulating particles) is, besides the magnetic field profile, one of the most easy to measure quantity that characterizes a magnetic hole [e.g., Winterhalter et al., 1994]. Given $n^{+}$from (4) and $\lambda$ which will be determined later using the pressure balance condition (12), $\eta$ must necessarily satisfy to the following condition

$$
\eta \equiv n^{-} / n^{+}=\left[1-(1-\lambda) n^{+}\right] \lambda / n^{+} .
$$

In fact, this expression is consistent with the requirement $n^{-} \lambda+n^{+}(1-\lambda)=1$ which ensures that the total number of particles in the system is conserved. Let us now compute the total number of particles that are trapped for a given field strength $\delta B^{+}$. This number, denoted $N_{\mathrm{tr}}$, includes all particles that do not verify the inequality (3). Thus

$$
\frac{N_{\mathrm{tr}}}{N_{0}}=\int_{\left|v_{0\|\|}\right|<v_{0 \perp} \sqrt{\delta B^{+}}} f_{0} d v_{0}^{3}=\left(\frac{\delta B^{+}}{R+\delta B^{+}}\right)^{1 / 2}
$$

where $N_{0}$ is the total number of particles in the system. The total number of circulating particles is then simply given by

$$
N_{\text {circ }}=N_{0}-N_{\text {tr }} \text {. }
$$

Let us now come to the determination of $\lambda$. We start by writing the pressure balance condition for our system. Given the simplicity of the configuration, we are considering here the latter reduces to a balance between the pressure (particle + field) in the high magnetic field region and the pressure in the low magnetic field region. Using the above normalization, this can be written in the following way

$$
p_{\perp, \mathrm{tot}}^{+}=p_{\perp, \mathrm{circ}}^{-}+p_{\perp, \mathrm{tr}}^{-}+\left(B^{-}\right)^{2} / \beta_{\|}
$$

where $p_{\perp, \text { circ }}^{-}$and $p_{\perp \text {,tr }}^{-}$are the pressure of the circulating and the trapped particles in the magnetic wells, respectively. In reality, since $B^{-}$is related to $\lambda$ through (2), (12) is nothing else but an equation for $\lambda$. However, in order to solve (12) we nced to determine the dependence on $\lambda$ of $p_{\perp, \text { circ }}^{-}$ and $p_{\perp, \mathrm{tr}}^{-}$. For this purpose we observe that the contribution to the pressure coming from one single particle is proportional to $B$ so that $p_{\perp, \text { circ }}^{-}$and $p_{\perp, \text { tr }}^{-}$are both proportional to $B^{-}$. On the other hand, the pressure of a given population changes proportionally to the spatial size of the domain occupied by the population. In order to make things simple we suppose that all the populations we are going to consider below are distributed uniformly at $t=0$. This is reasonable for two reasons. First, at $t=0$ the distribution of the sum of all proton populations is given by the distribution function (1). Second, the growth of the instability is slow compared to the time it takes for a particle to cross the system. The latter means that two particles with ncarly the same vclocity and the same position at time $t$ originate, in general, from two completely different positions at $t=0$; that is, the memory of the initial position is lost. Stated differently, one can say that a particle located near a given position at time $t$ was with the same probability located in the vicinity of any of the points in the interval $[0,1]$ at $t=0$. As a consequence, we 
may assume that the population of trapped particles covers uniformly the whole domain $[0,1]$ at $t=0$ but that this same population is confined to the domain $[0, \lambda]$ at time $t$. Summarizing these considerations, we conclude that the pressure contribution due to the trapped particles at time $t$ must be given by

$$
p_{\perp, \mathrm{tr}}^{-}=p_{0 \perp, \mathrm{tr}} B^{-} / \lambda
$$

where $p_{0 \perp, \text { tr }}$ is the trapped particles' pressure at $t=0$, that is,

$$
\begin{aligned}
& p_{0 \perp, \operatorname{tr}}=\int_{\left|v_{0\|\|}\right|<v_{0 \perp} \sqrt{\delta B^{+}}} v_{0 \perp}^{2} f_{0} 2 \pi v_{0 \perp} d v_{0 \perp} d v_{0 \|} \\
& p_{0 \perp, \mathrm{tr}}=\left(\frac{\delta B^{+}}{R+\delta B^{+}}\right)^{1 / 2}\left[\frac{3 R+2 \delta B^{+}}{2 R\left(R+\delta B^{+}\right)}\right] \text {. }
\end{aligned}
$$

An argument, similar to the one for trapped particles, applies to the circulating particles. Suppose that the circulating particles, located in the low magnetic field region at time $t$, cover a fraction $\alpha$ of the domain $[0,1]$ at $t=0$. According to the above discussion, $\alpha$ must be the same as the ratio of the number of circulating particles located in the magnetic well at time $t$ to the total number of circulating particles. Since the total number of particles which are located in the high-field region at time $t$ is $n^{+}(1-\lambda)$, one has $\alpha=\left[N_{\text {circ }}-n^{+}(1-\lambda)\right] / N_{\text {circ }}$ which leads to

$$
\begin{aligned}
& p_{\perp, \mathrm{circ}}^{-}=p_{0 \perp, \mathrm{circ}} \alpha B^{-} / \lambda \\
& p_{\perp, \mathrm{circ}}^{-}=\left(\frac{1}{R}-p_{0 \perp, \mathrm{tr}}\right) \frac{\alpha B^{-}}{\lambda} .
\end{aligned}
$$

We can now express (12) as a function of the known quantities $\beta_{\|}, R, \delta B_{\mathrm{sat}}^{+}$and the remaining unknown parameter $\lambda$. This leads to a polynomial equation of degree three for $\lambda$. Physically acceptable solutions must be real and in the interval $[0,1]$. Only two solutions, shown in Figure 5, satisfy these requirements in the domain in parameter space $\left(\beta_{\|}, R\right)$ below the mirror instability threshold curve and outside the shaded domain. Elsewhere (above the mirror instability threshold curve and in the shaded domain) the two solutions are complex so that no equilibrium solution exists there. In the domain where the two roots are real their value is generally smaller then 0.5 , suggesting that the mirror instability tends to form narrow low magnetic field regions rather than the opposite. This is in qualitative agreement with most obscrvations [Winterhalter et al., 1994]. However, even though observations seem to indicate that the nonlinear evolution of the mirror instability leads to narrow and deep holes in the magnetic field, it sometimes happens that the opposite situation is observed. One such case is illustrated by Figure 2a in the Leckband et al. [1995] paper. The figure shows large nonlinear fluctuations of the magnetic field observed in the Earth's magnetosheath. The fluctuations can be described as a more or less irregular sequence of narrow magnetic field spikes. Even this particular episode is consistent with the results of the model. In fact, the plasma $\beta$, measured by Leckband et al., was exceptionally large $(\beta \approx 30)$. If we put the value $\beta_{\|}=20$ in our model we find that the stable solution, shown in Figure 5a for the domain $\beta_{\|}<10$ predicts that $\lambda$ is larger than 0.5 , provided the initial temperature anisotropy is not too weak, that is $R \lessgtr 0.5$.

Let us come back to the discussion of (12). Even though the two physically acceptable solutions of (12) correspond to possible equilibrium solutions, only one is a stable solution (Figure 5a). Figure 5b corresponds to a solution with $\partial p_{\perp, \text { tot }}^{-} / \partial B^{-}<0$ (at saturation), that is, the total pressure and the magnetic pressure vary in antiphase in the low-field region. Thus only Figure 5a corresponds to a totally stable equilibrium for the final configuration. We note, however, that the two solutions become identical on the border of the shaded region and that they become both vanishing small as one approaches the instability threshold from below.

Figure 6 is a contour plot of the density ratio $\eta$ of the low to high magnetic field region densities (equation (9)). The figure shows that for reasonable anisotropies $R \gtrsim 0.4$ the values for $\eta$ do not exceed 2.5 which is consistent with

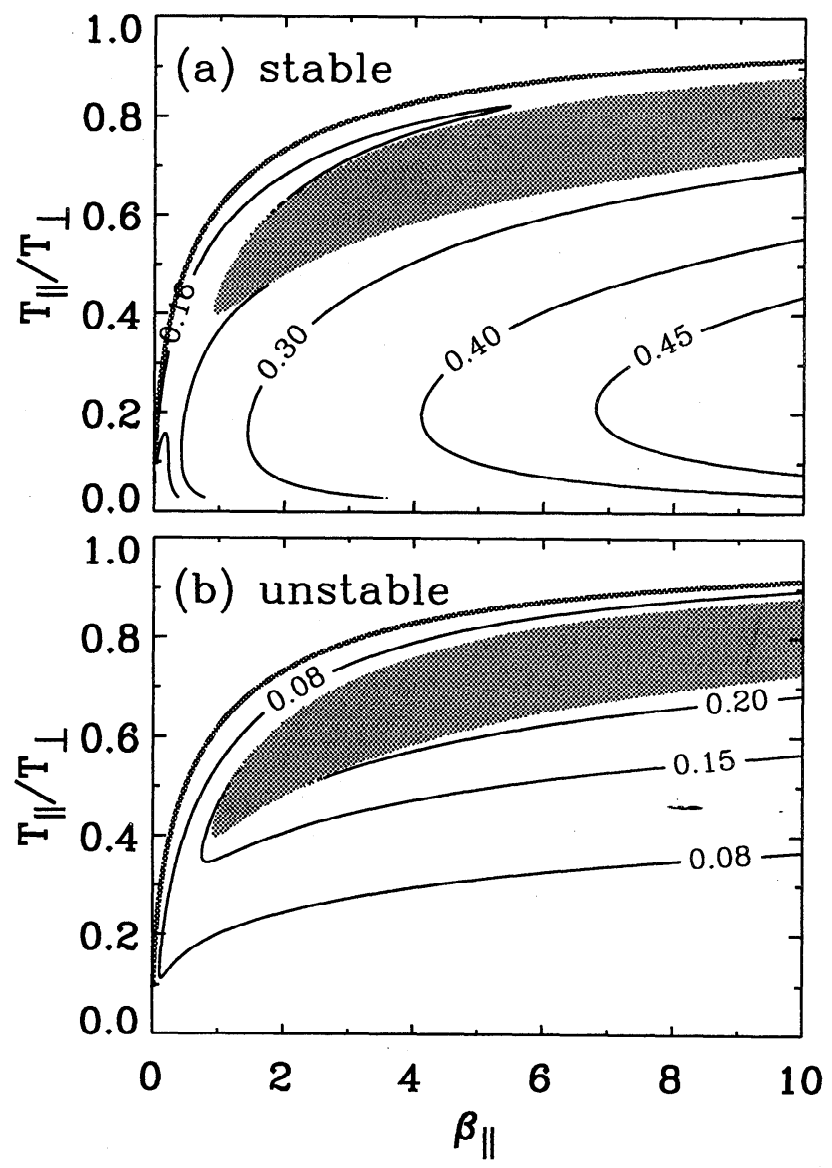

Figure 5. Contours for the two positive solutions $\lambda$ of (12). The solutions become complex conjugate above the mirror mode stability threshold and in the shaded region where no stable solutions exist. (a) Stable solution with $\partial p_{\perp, \text { tot }}^{-} / \partial B^{-}<0$. (b) Unstable solution with $\partial p_{\perp, \text { tot }}^{-} / \partial B^{-}>0$. 


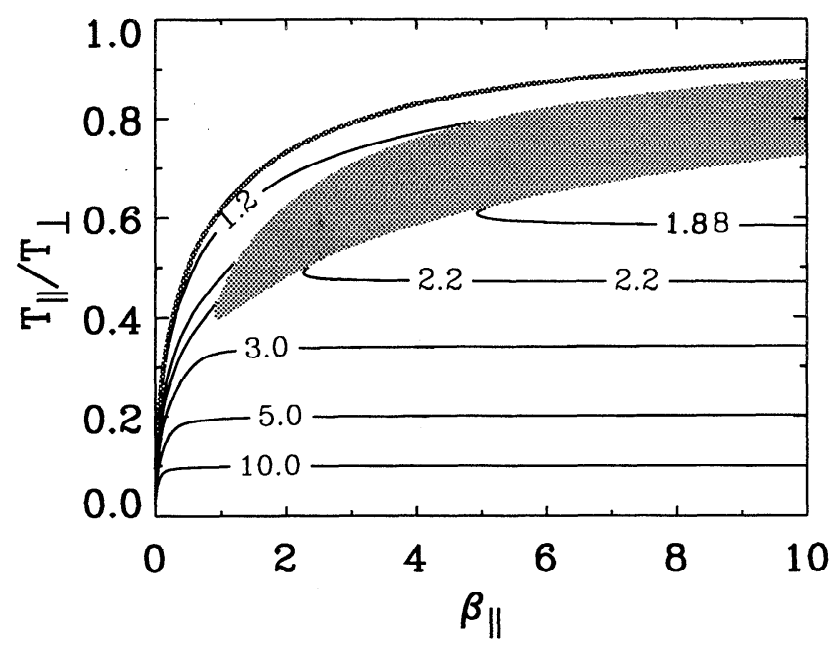

Figure 6. Contours of the density ratio $n^{-} / n^{+}$computed using (9) for the case where $\lambda$ is the stable solution of Figure 5 a.

the typical values observed in the solar wind by Winterhalter et al. [1994]. Concerning the fact that observed values for $\eta$ seem to be closer to unity than what may eventually suggest our model, it should be pointed out here that all the above results are valid in the case of a cold electron population such that its temperature $T_{e}$ is much smaller than the parallel proton temperature, that is, $T_{e} \ll T_{\|}$. Electron temperature effects have been shown to play an important role in the linear mirror instability [Pantellini and Schwartz, 1995]. In particular, a hot electron population significantly reduces the absolute value of the parallel compressibility (a measure of the density fluctuations compared to the magnetic field fluctuations) in a plasma with $T_{e} \gtrsim T_{\|}$. We therefore expect the electron temperature effects to act in the sense of reducing the value of $\eta$ shown in Figure 6. Still, it should be noted that even in the cold electrons framework it is possible to obtain relatively small values of $\eta$ associated with narrow and deep holes. For example, the case $\beta_{\|}=2$ and $R=0.65$ (weakly unstable) leads to the stable asymptotic state characterized by $\lambda_{\text {sat }}=0.17, \delta B_{\text {sat }}^{+}=0.12$ and $\delta B_{\text {sat }}^{-}=0.59$, which represents a noticeable magnetic hole, while the associated density ratio $\eta=1.34$ is rather small.

Let us conclude this section with a comment concerning the validity of the presented model. The most important restriction comes from the fact that the circulating particles are supposed to conserve energy. As already stated above, this cannot be true for particles located near the velocity space trapping boundary. Thus in cases where the number of circulating particles that do not conserve energy is large (e.g., in the case of fast growth of the instability), the modcl is certainly inappropriate. In order to estimate the domain of validity of the model, let us suppose that during the time of growth of the instability the maximum growth rate is $\gamma=2 \pi / t_{\text {growth }}$. This growth rate is likely to be the growth rate predicted by linear theory, as in the nonlinear phase of the instability growth tends to become smaller as the instability saturates. On the other hand, at saturation, in the highfield region, a circulating particle that conserves energy has a field-aligned velocity given by $v_{\|}=\sqrt{v_{0 \|}^{2}-v_{0 \perp}^{2} \delta B_{\mathrm{sat}}^{+}}$. If $v_{\|}$is large enough, so that the time it takes for the particles to cross the high-field region is shorter than the typical growth time $t_{\text {growth }}$, the assumption of energy conservation is a good approximation. This leads immediately to the condition $v_{\|}>\gamma(1-\lambda) /(2 \pi)$. Since linear theory provides a value for $\gamma$, but not for $\lambda$, we may use the more restrictive condition $\sqrt{v_{0\|\|}^{2}-v_{0 \perp}^{2} \delta B_{\text {sat }}^{+}}>\gamma /(2 \pi)$ which states that only particles with an initial parallel velocity within $\gamma /(2 \pi)$ from the separatrix do not conserve energy. Note that for small $\delta B_{\text {sat }}^{+}$the above condition is the same condition that distinguishes circulating from resonant particles in the linear mirror instability [Southwood and Kivelson, 1993].

\section{Discussion}

We have presented a model for the formation of stable nonpropagating structures in a collisionless magnetized plasma. The model is based on the fact that in a plasma with cold electrons and a bi-Maxwellian proton distribution with $T_{\perp}>T_{\|}$the total pressure responds in antiphase to a lowfrequency compressional perturbation of the magnetic field provided the criterion for the mirror instability is satisfied. Saturation is assumed to occur when the magnetic pressure and the total pressure of the plasma start varying in phase. The model is in fact a model of the nonlinear mirror instability where saturation is the consequence of particle trapping in the low magnetic field regions of the evolved structure.

The model is similar to the one proposed by Kivelson and Southwood [1996] in that the saturated state is one where the total pressure is the same for both the high and the low magnetic field region. The main difference stems from the fact that in our model a particular magnetic field profile, shown in Figure 1, has been chosen. Compared to the more general, but essentially qualitative, model of Kivelson and Southwood, our model has the advantage of providing quantitative estimates of the characteristics of the evolved structure (Figures 4 to 6).

The basic conclusion of the Kivelson and Southwood analysis is that cooling of the trapped particles, which is needed to reach pressure equilibrium, is likely to result from a mixture of betatron and Fermi deceleration. A quantitative estimate of the final state is not possible in their case-as the evolution of the instability does in general depend on the unknown temporal and spatial variations of the magnetic field profile. The simplifications in our model are such that the temporal evolution of the field becomes unimportant and the final structure can in gencral be computed unambiguously as a function of the initial plasma conditions only. Moreover, the detailed behavior of the trapped particles, which may be rather complicated, does not need to be known.

The main results can be summarized as follows. First of all, a simple expression for the saturation level of the nonlinear mirror instability is found. As shown in Figure 4, the saturation level generally increases with both $\beta_{\|}$and the temperature anisotropy $T_{\perp} / T_{\|}$. We also observe that a stable final solution can nearly always be found in the linearly un- 
stable domain except in the narrow shadowed domain shown in Figures 4-6. A more general treatment of the instability (allowing for structures other than the one shown in Figure 1) as the one discussed by Kivelson and Southwood [1996] may probably lead to a stable final state even in the shadowed domain shown in our figures. We have argued that finite electron temperature effects, that have been shown to be important in linear theory, may also significantly modify the final equilibrium configuration. As suggested by Kivelson and Southwood [1996], we observe that substantial cooling of the particles in the magnetic wells is generally necessary in order to reach the final equilibrium solution. Thus the evolved structure tends to be one where the low magnetic field regions are narrower than the high magnetic field regions unless the plasma $\beta$ is very high (i.e., $\beta \gtrsim 10$ ). Narrow wells also mean deep wells which seems to agrec with the majority of the observed magnetic holes wherein magnetic field intensities as low as $10 \%$ of the out of hole value are not unusual [e.g., Winterhalter et al., 1994]. The model suggests that the holes are narrowest in plasma that are marginally unstable and predicts that the particle density is always higher in the magnetic holes than in surrounding plasma, which is consistent with observation. Finally, since our results are independent of the amount of Fermi acceleration (or deceleration) that affects the trapped particles, we conclude that the most important mechanism leading to saturation is the betatron cooling of the trapped protons.

Further improvements of the model may be obtained rather easily by including electron temperature effects. The treatment of more general field profiles, compared to the one used here, may not be possible without making the model considerably more complicated. Particle simulations of the type published by McKean et al. [1992, 1993] may help in better understanding the details of the formation of magnetic holes whereas full particle simulations (including the electron dynamics) would be extremely useful in order to determine whether or not the high-frequency waves (for example, the so-called lion roars [Tsurutani et al., 1982]), frequently observed in magnetic holes, are effectively due to the electron cyclotron instability as suggested by Lee et al. [1987]. Particle simulations will also be needed to understand the effects on the formation of stable structure due to finite Larmor radius effects. Our model as well as the Kivelson and Southwood [1996] one are both based on the hypothesis of magnetic moment conservation for all particles. It is clear that such an approximation will fail in the case of strong instabilities where the most unstable wavelength becomes of the order of the proton Larmor radius with a typical growth rate comparable to the Larmor frequency. The complexity of the particles' motion in a field where the typical spatial and temporal scales are of the order of the Larmor scales is such that only numerical simulations may help farther under these conditions.

Acknowledgments. I thank R. J. MacDowall for useful comments. The Editor thanks D. Winterhalter and another referee for their assistance in evaluating this paper.

\section{References}

Anderson, B.J., and S.A. Fuselier, Magnetic pulsations from 0.1 to $40 \mathrm{~Hz}$ and associated plasma properties in the Earth's subsolar magnetosheath and depletion layer, J. Geophys. Res., 98, 1461, 1993.

Barnes, A., Collisionless damping of hydromagnetic waves, Phys. Fluids, 9, 1483, 1966.

Fitzenreiter, R.J., and L.F. Burlaga, Structure of current sheets in magnetic holes at $1 \mathrm{AU}$, J. Geophys. Res., 83, 5579, 1978.

Glassmeier, K.-H., U. Motschmann, C. Mazelle, F.M. Neubauer, K. Sauer, S.A. Fuselier, and M.H. Acuna, Mirror modes and fast magnetoacoustic waves near the magnetic pileup boundary of comet P/Halley, J. Geophys. Res., 98, 20955, 1993.

Hasegawa, A., Drift mirror instability in the magnetosphere, Phys. Fluids, 12, 2642, 1969.

Hubert, D., C. Perche, C.C. Harvey, C. Lacombe, and C.T. Russell, Observation of mirror waves downstream of a quasi-perpendicular shock, Geophys. Res. Lett., 16, 159, 1989.

Kaufmann, R.L., J.T. Horng, and A. Wolfe, Large-amplitude hydromagnetic waves in the inner magnetosheath, J. Geophys. Res., $75,4666,1970$.

Kivelson, M.G., and D.J. Southwood, Mirror instability, 2, The mechanism of nonlinear saturation, J. Geophys. Res., 101, 17365 , 1996.

Klein, L., and L.F. Burlaga, Interplanetary sector boundaries 19711973, J. Geophys. Res., 85, 2269, 1980.

Lacombe, C., F.G.E. Pantellini, D. Hubert, C.C. Harvey, A. Mangeney, G. Belmont, and C.T. Russell, Mirror and Alfvénic waves observed by ISEE1-2 during crossing of the Earth's bow shock, Ann. Geophys., 10, 772, 1992.

Leckband, J.A., D. Burgess, F.G.E. Pantellini, and S.J. Schwartz, Ion distributions associated with mirror waves in the Earth's magnetosheath, Adv. Space Res., 15, 345, 1995.

Lee, L.C., C.S. Wu, and C.P. Price, On the generation of magnetosheath lion roars, J. Geophys. Res., 92, 2343, 1987.

McKean, M.E., D. Winske, and S.P. Gary, Mirror and ion cyclotron anisotropy instabilities in the magnetosheath, J. Geophys. Res., 97, 19421, 1992.

McKean, M.E., S.P. Gary, and D. Winske, Kinetic physics of the mirror instability, J. Geophys. Res., 98, 21313, 1993.

Pantellini, F.G.E., and S.J. Schwartz, Electron temperature effects in the linear proton mirror instability, J. Geophys. Res., 100, $3539,1995$.

Price, C.P., D.W. Swift, and L.C. Lee, Numerical simulation of nonoscillatory mirror waves at the Earth's magnetosheath, $J$. Geophys. Res., 91, 101, 1986.

Russell, C.T., W. Riedler, K. Schwingenshuh, and Y. Yeroshenko, Mirror instability in the magnetosphere of Comet Halley, Geophys. Res. Lett., 14, 664, 1987.

Shapiro, V.D., and V.I. Shevchenko, Quasilinear theory of instability of a plasma with an anisotropic ion velocity distribution, Sov. Phys. JETP, Engl. Transl., 18, 1109, 1964.

Southwood, D.J., and M.G. Kivelson, Mirror instability, 1, The physical mechanism of linear instability, J. Geophys. Res., 98, 9181, 1993.

Tajiri, M., Propagation of hydromagnetic waves in collisionless plasma, 2, Kinetic approach, J. Phys. Soc. Jpn., 22, 1482, 1967.

Tsurutani, B.T., E.J. Smith, R.R. Anderson, K.W. Ogilvie, J.D. Scudder, D.N. Baker, and S.J. Bame, Lion roars and nonoscillatory drift mirror waves in the magnetosheath, J. Geophys. Res., 87, 6060, 1982.

Tsurutani, B.T., D.J. Southwood, E.J. Smith, and A. Balogh, Nonlinear magnetosonic waves and mirror mode structures in the March 1991 Ulysses interplanetary event, Geophys. Res. Lett., 19, 1267, 1992.

Turner, J.M., L.F. Burlaga, N.F. Ness, and J.F. Lemaire, Magnetic holes in the solar wind, J. Geophys. Res., 82, 1921, 1977.

Violante, L., M.B. Bavassano Cattaneo, G. Moreno, and J.D. Ri- 
chardson, Observations of mirror waves and plasma depletion layer upstream of Saturn's magnetopause, J. Geophys. Res., 100, $12047,1995$.

Winterhalter, D., M. Neugebauer, B.E. Goldstein, E.J. Smith, S.J. Bame, and A. Balogh, Ulysses field and plasma observations of magnetic holes in the solar wind and their relation to mirrormode structures, J. Geophys. Res., 99, 23371, 1994.
Filippo Pantellini, Département de Recherche Spatiale, Observatoire de Paris-Meudon, 5 Place Jules Janssen, 92195 Meudon

Cedex, France. (e-mail: Filippo.Pantellini@obspm.fr)

(Received February 17, 1997; revised August 15, 1997; accepted August 18, 1997.) 